\title{
Does increasing the arterial blood pressure at the end of a laparoscopic gastric bypass relates to the number of postoperative haemorrhages?
}

\author{
Cécile TENEY MD ${ }^{1,3}$, Jan MULIER MD PhD ${ }^{7}$, Bruno DILLEMANS MD ${ }^{2}$, Michel VAN DYCK MD ${ }^{3}$
}

${ }^{1}$ Dep. Anaesthesiology, AZ Sint Jan Brugge-Oostende, Belgium.

${ }^{2}$ Dep. General Surgery, AZ Sint Jan Brugge-Oostende, Belgium.

${ }^{3}$ Dep. Anaesthesiology Cliniques universitaires Saint Luc, UCL, Brussels, Belgium.

\section{Introduction}

From 2004 till $2008^{(1)}$ a large number of gastric bypass patients have had postoperative haemorrhages (92 haemorrhages on 2903 pt or 3,2\%) requiring frequent surgical revision (29 revisions or $0,9 \%$ ).

In 2009 we instructed to increase the systolic arterial blood pressure (SAP) to $140 \mathrm{mmHg}$ in every patient and reduced dramatically the number of haemorrhages $(3.2 \% \rightarrow 1 \%)$ and surgical revisions $(0.9 \% \rightarrow$ $0,1 \%)$ in 2010 . Other surgical mesures have also been taken over the years (staplers, surgical protocols,...)

The following years some postoperative haemorrhages remained.

\section{Questions}

Did these patients with haemorrhage have an insufficient increase regarding intraoperative blood pressure, did we treat their postoperative hypertension sufficiently, or was it related to other factors?

\section{Methods}

- Retrospective study of all gastric bypass operations in AZ Sint Jan.

- 5237 consecutive patients are included between 2011 until mid 2015

- The highest Systolic Arterial Pressure (SAP) reached before the end of the procedure and the highest SAP reached in the PACU is recorded. The ethical committee validated the retrospective analysis.

- A multivariate linear regression analysis is used to find the bleedingrelated factors; or insufficient SAP-increase or higher SAP-value in the PACU.

Total haemorrhagic complications and surgical revisions

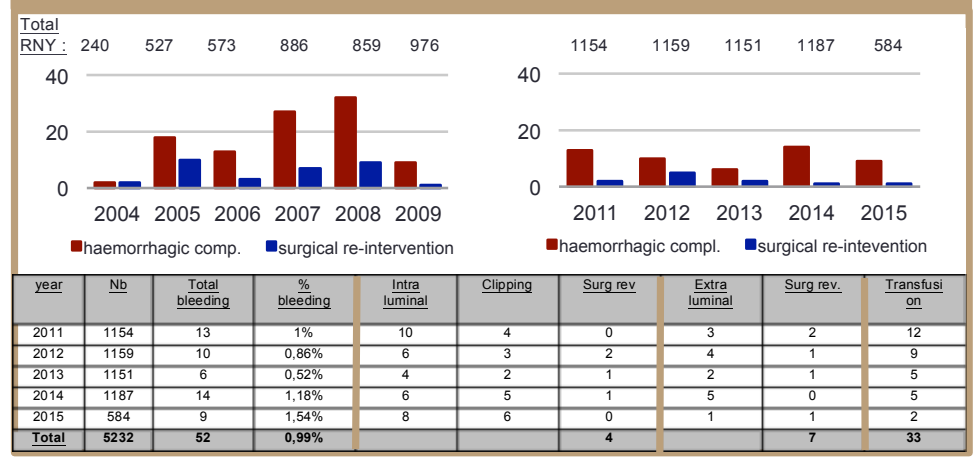

Demographic differences between patients with and without haemorrhagic complication

\begin{tabular}{|c|c|c|c|c|c|}
\hline \multicolumn{3}{|c|}{ Demographic differences } & \multicolumn{3}{|c|}{ Logistic regression analysis } \\
\hline & $\begin{array}{l}\text { Non bleeder: } \\
5182 \text { patients }\end{array}$ & $\begin{array}{c}\text { Bleeder } \\
57 \text { patients }\end{array}$ & Variable & Coefficient & $p$-value \\
\hline Age & $\begin{array}{c}41,563 \\
\pm 0,34\end{array}$ & $\begin{array}{c}46,845 \\
\pm 2,8\end{array}$ & $\begin{array}{c}\text { Age } \\
\text { (years) }\end{array}$ & 0,033 & 0,008 \\
\hline BMI & $\begin{array}{r}40,987 \\
\pm 0,154\end{array}$ & $\begin{array}{l}40,765 \\
\pm 1,751\end{array}$ & $\begin{array}{c}\text { BMI } \\
\left(\mathbf{k g} / \mathbf{m}^{2}\right)\end{array}$ & $-0,001$ & 0,953 \\
\hline Diabetes & $\begin{array}{c}673 \\
(12,99 \%)\end{array}$ & $\begin{array}{c}7 \\
(12.8 \%)\end{array}$ & $\begin{array}{c}\text { Diabetes } \\
(\text { yes }=1)\end{array}$ & $-0,388$ & 0,368 \\
\hline Hypertension & 1595 & $\frac{(12,0 \%)}{18}$ & $\begin{array}{c}\text { Hypertension } \\
(\text { yes }=1)\end{array}$ & $-0,496$ & 0,220 \\
\hline OSAS & & $\frac{(31,58 \%)}{29}$ & $\begin{array}{l}\text { OSAS } \\
\text { (yes=1-) }\end{array}$ & 0,196 & 0,481 \\
\hline Gender (male) & $\begin{array}{c}(45,08 \%) \\
1436\end{array}$ & $\begin{array}{c}(50,88 \%) \\
28\end{array}$ & $\begin{array}{l}\text { Gender } \\
(\text { male=1) }\end{array}$ & 1,018 & $<0,001^{*}$ \\
\hline & $(27,71 \%)$ & $(49,12 \%)$ & Xarelto - & $-0,216$ & 0,770 \\
\hline $\begin{array}{l}\text { Xarelto- } \\
\text { Asaflow }\end{array}$ & $\begin{array}{l}154 \\
(2,9 \%)\end{array}$ & $\begin{array}{c}4 \\
(7 \%)\end{array}$ & $\begin{array}{l}\text { Asaflow } \\
(y e s=1)\end{array}$ & & \\
\hline
\end{tabular}

Maximum blood pressure at end-operation and PACU in patients with or without haemorrhagic complications

Only $30 \%$ of the patients reached $140 \mathrm{mmHg}$ or more and $20 \%$ did not reach $120 \mathrm{mmHg}$ !

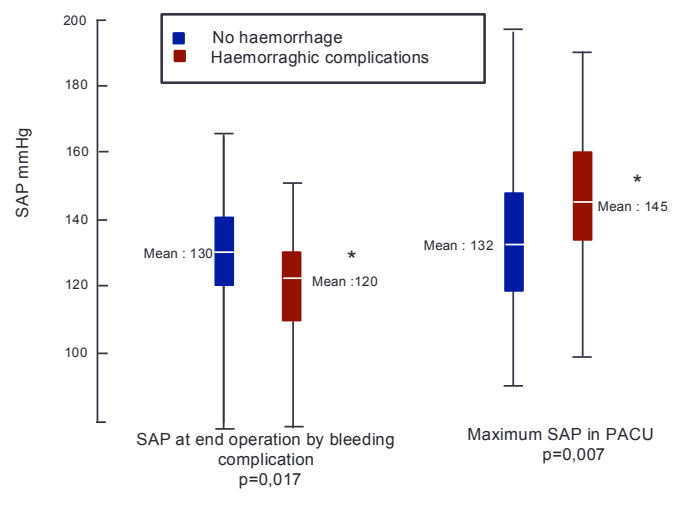

In a linear regression analysis

Profile of patients with a higher SAP in the PACU

\begin{tabular}{|c|c|c|c|c|c|}
\hline \multicolumn{3}{|c|}{ Demographic differences } & \multicolumn{3}{|c|}{ Logistic regression analysis } \\
\hline & $\begin{array}{c}\text { Postop } \\
\text { normotension }\end{array}$ & $\begin{array}{c}\text { Postop } \\
\text { hypertension }\end{array}$ & Variable & Coefficient & P-Value \\
\hline \multirow{2}{*}{ Age (years) } & $\leq 130 \mathrm{mmHg}$ & $>130 \mathrm{mmHg}$ & Age (years) & $-0,005$ & 0,949 \\
\hline & $\begin{aligned} 41,1 \\
\pm 0,325 \\
\pm\end{aligned}$ & $\begin{array}{l}42,81 \\
\pm 1,441 \\
\end{array}$ & Sufenta per op & 0,609 & $<0,001$ * \\
\hline $\begin{array}{c}\text { Total morphine } \\
\text { equivalent post } \\
\text { op (mg) }\end{array}$ & $\begin{array}{l}6,218 \\
\pm 0,349\end{array}$ & $\begin{array}{l}17,868 \\
\pm 1,752\end{array}$ & $\begin{array}{l}\text { (mcg) } \\
\text { Total morphine } \\
\text { equivalent post }\end{array}$ & 0,254 & 0,001 * \\
\hline $\begin{array}{c}\text { BMI } \\
\left(\mathrm{kg} / \mathrm{m}^{2}\right)\end{array}$ & $\begin{array}{l}41,5 \\
\pm 4,629\end{array}$ & $\begin{array}{l}40,478 \\
\pm 1,795\end{array}$ & $\begin{array}{l}\text { op (mg) } \\
\text { Hypertension }\end{array}$ & 4,899 & $0,011^{*}$ \\
\hline \multirow{2}{*}{$\begin{array}{c}\text { Sufenta } \\
\text { (mcg) }\end{array}$} & 6,637 & 15,583 & $($ yes $=1)$ & & \\
\hline & $\pm 0,425$ & $\pm 1,507$ & Gender & 4,292 & 0,019 \\
\hline $\begin{array}{l}\text { Diabetes } \\
\text { (yes =1) }\end{array}$ & $\begin{array}{c}641 \\
(11,36 \%)\end{array}$ & $\begin{array}{c}43 \\
(13,60 \%)\end{array}$ & & & \\
\hline $\begin{array}{l}\text { Hypertension } \\
\text { (yes=1) }\end{array}$ & $\begin{array}{c}1514 \\
(26,84 \%)\end{array}$ & $\begin{array}{c}106 \\
(33,54 \%)\end{array}$ & & & \\
\hline $\begin{array}{c}\text { OSAS } \\
(\text { yes }=1)\end{array}$ & $\begin{array}{c}2103 \\
(37,29 \%)\end{array}$ & $\begin{array}{c}163 \\
(51,58 \%)\end{array}$ & & & \\
\hline $\begin{array}{l}\text { Gender } \\
\text { (male=1) }\end{array}$ & $\begin{array}{c}1511 \\
(26,79 \%)\end{array}$ & $\begin{array}{c}107 \\
(33,86 \%)\end{array}$ & & & \\
\hline Total : & 5640 patients & 316 patients & & & \\
\hline
\end{tabular}

$\underline{\text { Conclusion }}$

The aim to increase the SAP to $140 \mathrm{mmHg}$ at the end of a laparoscopic gastric bypass is not achieved for all patient. More haemorrhages happen persistant low intra operative blood pressure and high postoperative blood pressure. Opioids use intra and post operative reduces optimal SAP, so it increases haemorragic complications.
1. Standardization of the Fully Stapled Laparoscopic Roux-en-Y Gastric Bypass for Obesity Reduces Early Immediate Postoperative Morbidity and Mortality: A Single Center Study on 2606 Patients. B Dillemans et al 2009 Obes Surg. 